Check for updates

Cite this: J. Mater. Chem. A, 2018, 6 , 12794

DOI: $10.1039 / \mathrm{c} 8 \operatorname{ta9} 0136 \mathrm{~h}$

www.rsc.org/MaterialsA

\section{Correction: Water splitting to hydrogen over epitaxially grown InGaN nanowires on a metallic titanium/silicon template: reduced interfacial transfer resistance and improved stability to hydrogen}

\author{
Mohamed Ebaid, ${ }^{a}$ Jung-Wook Min, ${ }^{a}$ Chao Zhao, ${ }^{a}$ Tien Khee $\mathrm{Ng},{ }^{a}$ Hicham Idriss ${ }^{* b}$ \\ and Boon S. Ooi ${ }^{\star a}$
}

Correction for 'Water splitting to hydrogen over epitaxially grown InGaN nanowires on a metallic titanium/ silicon template: reduced interfacial transfer resistance and improved stability to hydrogen' by Mohamed Ebaid et al., J. Mater. Chem. A, 2018, 6, 6922-6930.

The authors regret an error in the ABPE calculation, arising from the conversion from Ag/AgCl potential to RHE. This does not affect the main claims and arguments in the article.

The corrected text, on page 6927 of the originating article, should read as follows:

"The InGaN NWs grown on Ti achieved an ABPE of $0.23 \%$ at $0.5 \mathrm{~V} v s$. RHE compared to only $0.01 \%$ at $0.86 \mathrm{~V} v s$. RHE for those grown on the Si-substrate. Hence, an approximately 23 times increase in power conversion efficiency was achieved by using metallic Ti as a charge extraction/collection interlayer."

The corrected version of Fig. 4 is shown below (Fig. 4(b) has been corrected; the other panels remain unchanged):
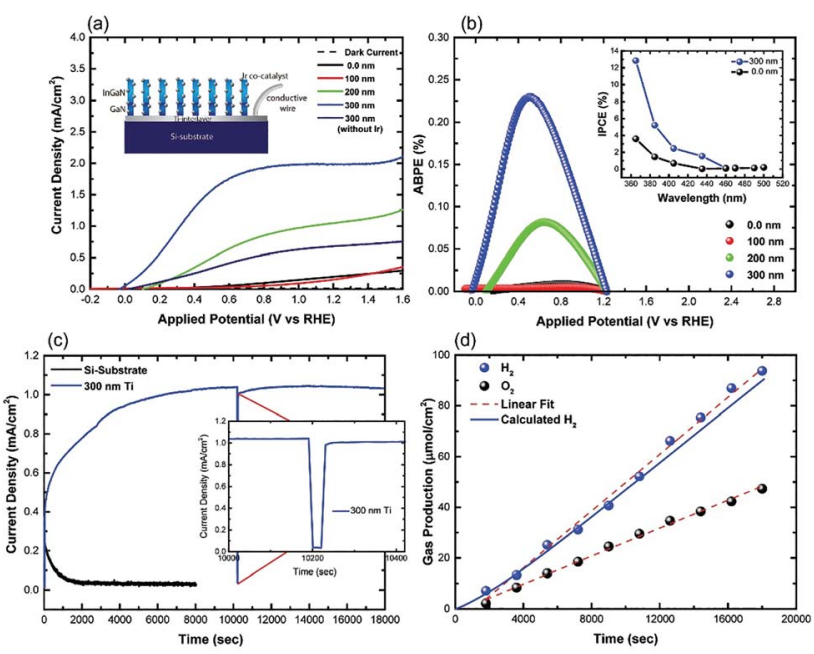

Fig. 4 PEC performance of InGaN NWs grown on Ti as compared to those on Si. (a) LSV of InGaN NWs under dark and light conditions. (b) ABPE of InGaN NWs as a function of Ti-thickness. The inset is the IPCE of InGaN NWs grown on $300 \mathrm{~nm}$ Ti as compared to those grown directly on Si. (c) Chronoamperometric test of the InGaN NWs on Ti and Si. (d) The gas evolution measured during the chronoamperometric test shown in (c). The light intensity was fixed at approximately $500 \mathrm{~mW} \mathrm{~cm}^{-2}$ during all the PEC measurements.

The Royal Society of Chemistry apologises for these errors and any consequent inconvenience to authors and readers. 